\title{
Survival analyses and immunohistochemical study of primary signet ring cell carcinoma of the lung adenocarcinoma
}

\author{
Chunji Chen ${ }^{1 \#}$, Lei Wang ${ }^{2 \#}$, Chang Gu ${ }^{3 \#}$, Yiyang Wang ${ }^{1}$, Xufeng Pan ${ }^{1}$, Shijie Fu ${ }^{1}$, Jun Yang ${ }^{1}$, Rui Wang ${ }^{1}$ \\ ${ }^{1}$ Department of Thoracic Surgery, ${ }^{2}$ Department of Ultrasound in Medicine, Shanghai Chest Hospital, Shanghai Jiao Tong University, Shanghai \\ 200030, China; ${ }^{3}$ Department of Thoracic Surgery, Shanghai Pulmonary Hospital, Tongji University School of Medicine, Shanghai 200433, China \\ Contributions: (I) Conception and design: R Wang, C Chen; (II) Administrative support: R Wang, S Fu; (III) Provision of study materials or patients: \\ J Yang, S Fu, R Wang; (IV) Collection and assembly of data: C Chen, C Gu; (V) Data analysis and interpretation: C Chen, C Gu, Y Wang; (VI) \\ Manuscript writing: All authors; (VII) Final approval of manuscript: All authors. \\ \#These authors contributed equally to this work. \\ Correspondence to: Rui Wang; Jun Yang. Department of Thoracic Surgery, Shanghai Chest Hospital, Shanghai Jiao Tong University, 241 West \\ Huaihai Road, Shanghai 200030, China. Email: rui_wang788@163.com; YJ_yangjun@hotmail.cn.
}

Background: The clinicopathological features, immunohistochemical (IHC) characteristics and survival outcomes of primary lung adenocarcinoma with signet ring cells (LAdSRCs) component were analyzed.

Methods: A retrospective analysis of primary LAdSRCs consecutively collected from 2010 to 2014 was performed and compared with lung adenocarcinoma (ADC). We investigated the survival outcomes and the expression of cytokeratin 7 (CK7), CK20, thyroid transcription factor-1 (TTF-1) and villin in these 35 cases of primary LAdSRCs.

Results: We identified 1,715 patients in total, 35 (2.0\%) of whom had signet ring cell (SRC) component. Excepting for 2 cases without IHC information, a CK profile (CK7+/CK20-) were identified in 69.7\% (23/33) of LAdSRC; 22 (66.7\%) of 33 LAdSRCs exhibited positive TTF-1 expression, but no other TTF-1 positive was found in signet ring cell carcinoma (SRCC) of other organs, and villin was not identified in these 33 cases. Age, sex, tumor size, surgery resection, smoking history, tumor stage, nodal stage, predominant histology subtype, pathology and adjuvant therapy were all significant predictors of relapse-free survival (RFS) and overall survival (OS) in univariable analysis. Compared to lung ADC, the lung ADCs with SRC component had an inferior prognosis. In multivariable analysis, sex, tumor size, tumor stage, nodal stage, predominant histology subtype and adjuvant therapy were still significant predictors for both RFS and OS while the pathology [RFS: hazard ratio (HR), 0.918; 95\% confidence interval (CI), 0.581-1.492; $\mathrm{P}=0.712$; OS: HR, 1.392; 95\% CI, 0.820-2.364; $\mathrm{P}=0.221$, respectively] was not.

Conclusions: Univariable analysis revealed primary LAdSRC is a rare ADC subtype with a worse prognosis compared with ADC. The high percentage expression of TTF-1 and immunostaining profiles CK7+/CK20- in primary LAdSRC is significant to identify the source of SRCCs.

Keywords: Signet-ring carcinoma of the lung; prognosis; immunohistochemistry

Submitted Oct 17, 2018. Accepted for publication Aug 08, 2019.

doi: $10.21037 /$ tcr.2019.11.54

View this article at: http://dx.doi.org/10.21037/tcr.2019.11.54 


\section{Introduction}

Lung cancer is the third most common malignancy in the world, leading to significant mortality and morbidity (1). Adenocarcinomas (ADCs) account for the majority of their pathological types and the incidence of them is now still rising $(2,3)$. Primary lung ADC with signet ring cell (SRC) is a rare subtype of lung ADC, which was originally described by Kish et al. in 1989 (4) and lung ADC with SRC was considered to be a unique subtype of lung cancer in the 2004 WHO lung ADC classification, which was designated as SRC ADC (5). However, in the 2015 WHO lung cancer classification, lung ADC with signet ring cells (LAdSRCs) are viewed as a cytologic change that may occur in association with various histological patterns rather than as a separate subtype, as they do not have a prognostic significance $(2,6)$. The poor prognosis that had been associated with LAdSRCs can be attributed to its strong correlation with solid subtype (7-9).

Signet ring cell carcinoma (SRCC) can occur in a variety of organs, but the incidence of SRCCs as a primary lung tumor was lower, ranging from $0.14 \%$ to $1.9 \%$ in the reported series $(2,10)$. Primary LAdSRCs and metastatic LAdSRCs have different management and treatment, and have different prognostic features. How to distinguish them is especially important. Several recent immunohistochemical (IHC) studies have shown that thyroid transcription factor-1 (TTF-1) was a tissue-specific transcription factor expressed almost exclusively in thyroid tissue and different tumors of the lung $(11,12)$. Simultaneously, immunostaining profiles of cytokeratin 7 (CK7) and CK20 have recently been used to identify SRC from different organ original such as breast, stomach and colon $(13,14)$. Therefore, CK7 and CK20 are important for the identification of primary LAdSRCs and metastatic LAdSRCs.

To date, only a few series of cases of primary SRC in the lung have been reported in the literature (7,15-17). Compared with lung ADC, the clinicopathological features and survival outcome of primary lung SRC were still undetermined. We investigated lung ADC with SRC components and compared the clinicopathological and IHC features of these 35 LAdSRCs and 1,680 lung ADCs.

\section{Methods}

The institutional review board of Shanghai Chest Hospital supported this study [KS(Y)1668]. Patients with lung ADC underwent surgical resection between 2010 and 2014 were all identified. Patients with history of malignancies within 5 years (including metastatic tumors in lung), and distant metastases were ineligible for the analysis; atypical adenomatous hyperplasia (AAH), adenocarcinoma in situ (AIS) and minimally invasive adenocarcinoma (MIA) were non-invasive lesions and free from lymph node (LN) metastasis. Thus, patients with these lesions were also excluded. All the patients received preoperative assessments including chest CT scanning, abdominal CT or ultrasound, brain CT scanning or MRI and radionuclide bone scanning, to confirm the diagnosis of primary lung tumor. Positron emission tomography (PET)-CT was an optimal substitution if necessary. The postoperative pathological staging of all patients was based on the $8^{\text {th }}$ edition of tumornode-metastasis (TNM) staging system.

The resected lung specimens and LNs were fixed in 10\% formalin and then embedded in paraffin. Subsequently, the hematoxylin and eosin (HE)-stained sections were evaluated microscopically by experienced pulmonary pathologists in our hospital. Diagnosis of SRC was determined by histological evaluation. SRC was a mucinsecreting ADC that was rich in cytoplasmic mucin and pushes the nucleus to the side of the cell. IHC studies were performed on formalin-fixed, paraffin-embedded tissue sections using the avidin-biotin-peroxidase complex method in a Dako Autostainer (Dako, Carpinteria, CA, USA). The pathological results and immunohistochemistry results of the specimens were provided by the Pathology Department of Shanghai Chest Hospital.

After the exclusion, a total of 1,823 patients were all primary lung ADC, including 35 LAdSRCs and 1,788 ADCs. Of the 1,823 lung ADCs, 35 patients were excluded for receiving neoadjuvant chemotherapy and 73 patients were excluded because they were lost to follow-up. The remaining 1,715 patients including 35 LAdSRCs and 1,680 ADCs were included in the study. All the patients were followed up through out-patient clinic or telephone every 3 months for the first year after surgery, every 6 months for the next 3 years and then annually.

\section{Statistical analysis}

All the clinicopathologic data and distributions of survival were analyzed by SPSS 23.0 software package (SPSS Inc., Chicago, IL, USA) and Prism 5 (Graphpad Software Inc., La Jolla, CA, USA). The curves of relapse-free survival (RFS) and overall survival (OS), as well as the comparisons, were calculated by Kaplan-Meier survival curves and 
the log-rank test. Two-sided $\mathrm{P}<0.05$ was set as statistical significance in this study.

\section{Results}

\section{Clinicopathologic features}

The characteristics are all listed in Table 1. Of those 1,715 patients included in the study, there were 718 males (41.9\%) and 997 females (58.1\%), with an average age of 59.5 (range from 22 to 84 ) years. We found that several clinicopathologic characteristics significantly differed between LAdSRCs and ADCs. The LAdSRCs show more male $(\mathrm{P}=0.011)$, more advanced disease stage $(\mathrm{P}<0.001)$, larger tumor size $(\mathrm{P}=0.032)$ and higher nodal stage $(\mathrm{P}<0.001)$.

The clinical details of 35 LAdSRCs are listed in Table 2. As for the tumor stage, 18 patients had pathologic stage (p-stage) III tumors, 13 patients had p-stage I tumors and 4 patients had p-stage IV tumors. In terms of predominant pathological subtypes, LAdSRCs was more common in solid subtype when compared with ADCs ( $37.1 \%$ vs. $9.3 \%, \mathrm{P}<0.001)$ and $45.7 \%(16 / 35)$ of them contained solid components. There were no significant differences in the age and smoking history.

\section{Survival analysis}

The median follow-up time and the median survival time of these patients were 62.4 and 40.8 months, respectively. Univariable analysis (Table 3) revealed that age, sex, tumor stage, tumor size, nodal stage, surgery resection, predominant histology subtype, adjuvant therapy, smoking history and SRC component were all significant predictors of RFS and OS while the sex, tumor stage, tumor size, nodal stage, predominant histology subtype and adjuvant therapy were still significant predictors in multivariable analysis (Table 4).

During the follow-up, 22 (62.9\%) LAdSRCs and 435 (25.9\%) lung ADCs experienced a relapse, and finally 17 (48.6\%) and 235 (14.0\%) patients died, respectively. There were significant differences in RFS and OS for lung ADCs with SRC component compared with those lung ADCs (Figures 1,2).

\section{Immunobistochemistry study}

The Table 2 summarize the results of immunostaining.
Except for two cases (No. 31 and No. 34) of missing IHC information, 22 of the 33 LAdSRCs (66.7\%) were TTF-1 positive; 23 of 33 LAdSRCs (69.7\%) expressed a CK profile of CK7+ or CK20-, and $2(6 \%)$ were positive for both CK7 and CK20. However, villin was not identified in these 33 cases.

\section{Discussion}

Since Kish et al. (4) originally reported 5 cases of 5,500 lung ADC patients with LAdSRC $(0.14 \%)$, there were sporadic reports on it $(4,5,10,16,18-21)$. Although the $2015 \mathrm{WHO}$ lung tumor classification discontinued the separate subtype of LAdSRCs and defined SRC as a type of cytologic characteristics (6), there has been no reduction in the focus on LAdSRCs.

In previously reported cases, the incidence of primary SRCC in the lung was $0.14 \%$ to $1.9 \%(2,10)$. In our study, 35 cases $(2.0 \%)$ of primary LAdSRCs were seen among 1,715 cases of surgically resected primary lung ADCs, which was basically consistent with previous research. In the case described by Kish et al., the proportion of SRCC components of primary LAdSRCs was defined as $10 \%$ to $50 \%$ and we defined LAdSRCs as tumor with SRC component in at least $5 \%$ of the tumor by light microscope according to the $2015 \mathrm{WHO}$ classification.

The 35 cases of primary LAdSRCs with an average age of 57.1 (range from 35 to 75) years. Compared with lung ADC, lung ADC with SRC component has worse RFS $(\mathrm{P}<0.001)$ and $\mathrm{OS}(\mathrm{P}<0.001)$ (Figures 1,2). This finding was in agreement with Tsuta et al. (7) who found 39 primary LAdSRCs and 20 cases (51.3\%) having died by the end of the study. A population-based epidemiologic study of 262 Cases conducted by $\mathrm{Ou}$ and colleagues (8) also indicated that primary LAdSRCs carry a worse prognosis when compared to ADCs. However, some previous reports indicated that the poor prognosis associated with LAdSRCs can be attributed to its strong association with solid subtypes (7-9). Our study also revealed that LAdSRCs are more common in solid subtype when compared with ADCs $(37.1 \%$ vs. 9.3\%, $\mathrm{P}<0.001)$. Furthermore, we divided LAdSRCs into two groups based on whether they contained solid components, and performed a survival analysis with ADCs and the results showed that the RFS and OS of the two groups were significantly worse than the ADCs $(\mathrm{P}=0.0026$ and $\mathrm{P}=0.0002$, respectively) (Figures S1,S2). This result suggested that perhaps SRC itself was a poor prognostic factor, but not the strong 
Table 1 Clinical characteristics of patients with LAdSRCs and ADC

\begin{tabular}{|c|c|c|c|c|c|}
\hline Characteristics & \multicolumn{2}{|c|}{ LAdSRCs (N=35) } & \multicolumn{2}{|c|}{ ADCs $(N=1,680)$} & $\mathrm{P}$ \\
\hline Age, years & & & & & 0.582 \\
\hline$<60$ & 19 & 54.3 & 833 & 49.6 & \\
\hline$\geq 60$ & 16 & 45.7 & 847 & 50.4 & \\
\hline Male & 22 & 62.9 & 696 & 41.4 & \\
\hline Female & 13 & 37.1 & 984 & 58.6 & \\
\hline Stage & & & & & $<0.001$ \\
\hline I-II & 13 & 37.1 & 1,426 & 84.9 & \\
\hline$\leq 3$ & 25 & 71.4 & 1,453 & 86.5 & \\
\hline$>3, \leq 7$ & 9 & 25.7 & 212 & 12.6 & \\
\hline$>7$ & 1 & 2.9 & 15 & 0.9 & \\
\hline $\mathrm{N}$ stage & & & & & $<0.001$ \\
\hline NO & 16 & 45.7 & 1,380 & 82.1 & \\
\hline N1 & 1 & 2.9 & 91 & 5.4 & \\
\hline $\mathrm{N} 2$ & 18 & 51.4 & 209 & 12.4 & \\
\hline Surgery resection & & & & & 0.452 \\
\hline $\mathrm{M}$ or $\mathrm{S}$ & 13 & 37.1 & 157 & 9.3 & \\
\hline Variant & 8 & 22.9 & 92 & 5.5 & \\
\hline Adjuvant therapy & & & & & $<0.001$ \\
\hline Ever & 27 & 77.1 & 303 & 18.0 & \\
\hline Never & 8 & 22.9 & 1,377 & 82.0 & \\
\hline Smoking history & & & & & 0.696 \\
\hline Ever & 14 & 40.0 & 618 & 36.8 & \\
\hline Never & 21 & 60.0 & 1,062 & 63.2 & \\
\hline
\end{tabular}

LAdSRC, lung adenocarcinoma with signet ring cell; ADC, adenocarcinoma; L, lepidic; A, acinar; P, papillary, M, micropapillary; S, solid. 
Table 2 Clinical details of 35 lung ADCs with clear cell component

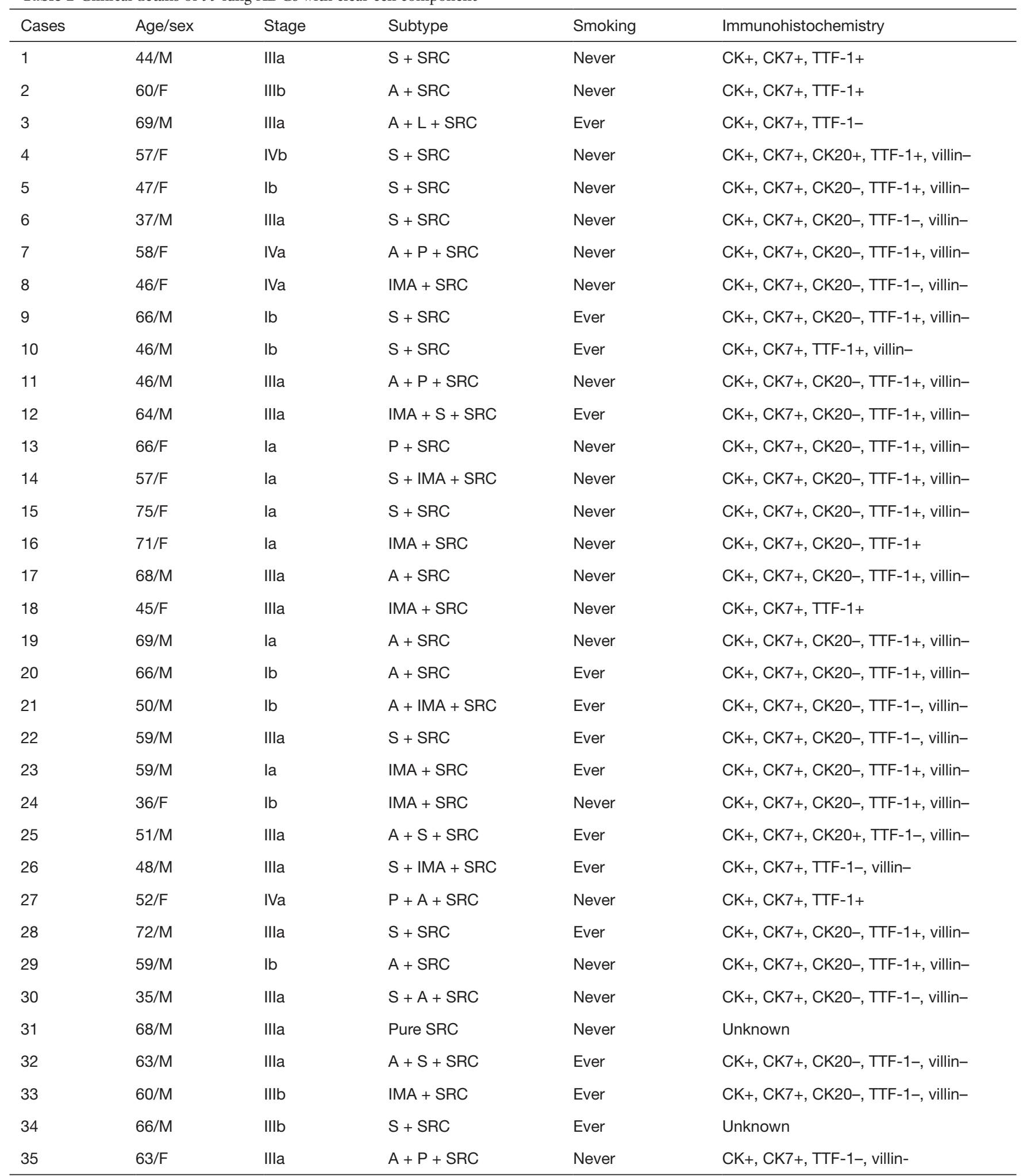

ADC, adenocarcinoma; M, male; F, female; A, acinar; P, papillary; S, solid; L, lepidic; IMA, invasion mucinous adenocarcinoma; SRC, signet ring cell; CK, cytokeratin; TTF-1, thyroid transcription factor-1. 
Table 3 Univariable analyses for RFS and OS in patients with resected lung ADC

\begin{tabular}{|c|c|c|c|c|c|c|}
\hline Characteristics & \multicolumn{3}{|c|}{ RFS } & \multicolumn{3}{|c|}{ OS } \\
\hline Age, years & 0.598 & $0.489-0.736$ & $<0.001$ & 0.697 & $0.586-0.921$ & $<0.001$ \\
\hline Sex & 1.401 & $1.166-1.684$ & $<0.001$ & 1.531 & $1.195-1.961$ & 0.001 \\
\hline Stage & 2.895 & $2.635-3.181$ & $<0.001$ & 2.960 & $2.613-3.353$ & $<0.001$ \\
\hline $\mathrm{N}$ stage & 2.898 & $2.629-3.196$ & $<0.001$ & 2.994 & $2.631-3.406$ & $<0.001$ \\
\hline Surgery resection & 1.694 & $1.245-2.305$ & 0.001 & 1.549 & $1.016-2.362$ & 0.042 \\
\hline Predominant histology subtype & 2.210 & $1.565-3.123$ & $<0.001$ & 2.531 & $1.648-3.888$ & $<0.001$ \\
\hline Adjuvant therapy & 1.244 & $1.007-1.538$ & 0.043 & 1.449 & $1.218-1.723$ & $<0.001$ \\
\hline
\end{tabular}

RFS, relapse-free survival; OS, overall survival; $\mathrm{ADC}$, adenocarcinoma; HR, hazard ratio; $\mathrm{Cl}$, confidence interval.

Table 4 Multivariable analyses of RFS and OS in patients with resected lung ADC

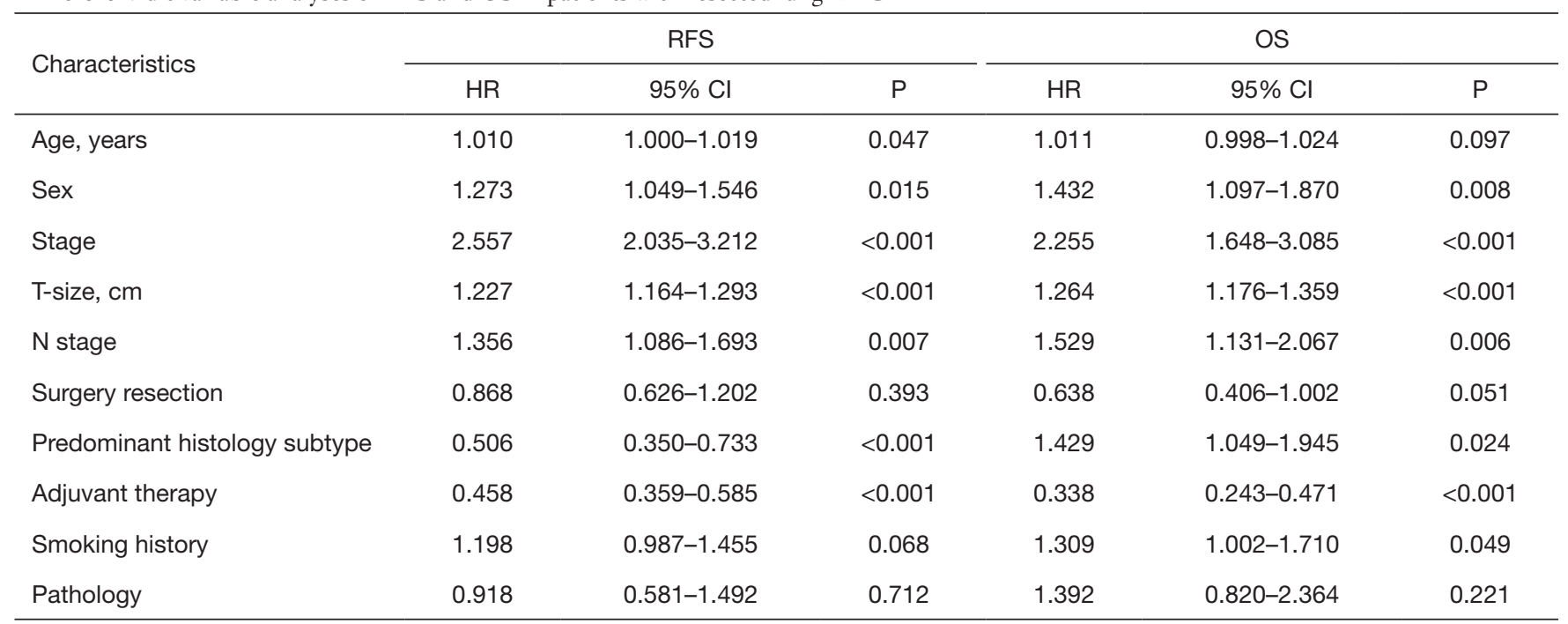

RFS, relapse-free survival; OS, overall survival; ADC, adenocarcinoma; HR, hazard ratio; $\mathrm{Cl}$, confidence interval.

association with solid subtype. SRC was only acted as a significant predictor of survival in univariate analysis (Table 3), but not in multivariate analysis (Table 4). The reason may be the fact that those lung ADCs with SRC component were associated with advanced disease stage $(\mathrm{P}<0.001)$ (stage III/IV, 62.9\%, Table 1).

By analyzing the IHC results of these 35 cases, we found some common features. Except for two cases without IHC information (Table 2), 22 of the 33 LAdSRCs (66.7\%) are
TTF-1 positive; 23 of 33 LAdSRCs (69.7\%) show a CK profile of $\mathrm{CK} 7+$ or CK20-, and $2(6 \%)$ expressed both CK7+ and CK20+. However, villin was not identified in these 33 cases. TTF-1 is a $38 \mathrm{kDa}$ nuclear protein member of the homologous gene transcription factor NKX2 family which was encoded by a single locus (22). Several recent IHC studies revealed that TTF-1 was a tissue-specific transcription factor expressed almost exclusively in thyroid tissue and different tumors of the lung, and it shown that it 


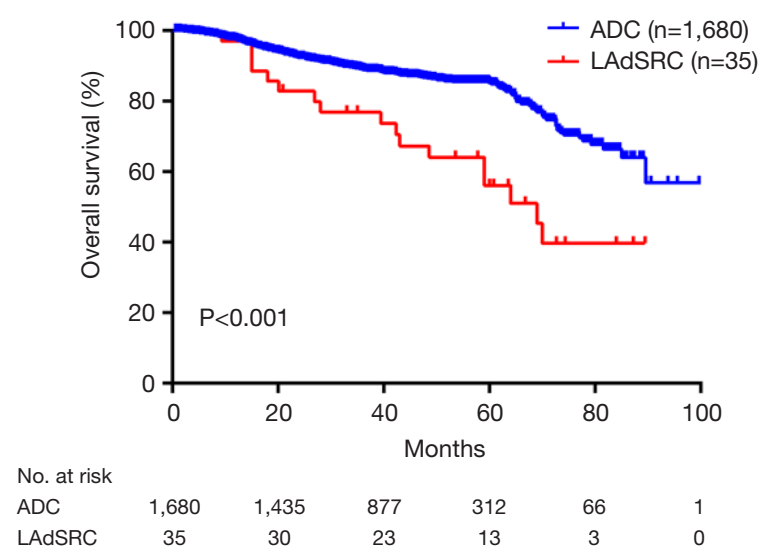

Figure 1 Kaplan-Meier survival curves for RFS according to LAdSRCs and lung ADCs. RFS, relapse-free survival; LAdSRC, lung adenocarcinoma with signet ring cell; ADC, adenocarcinoma.

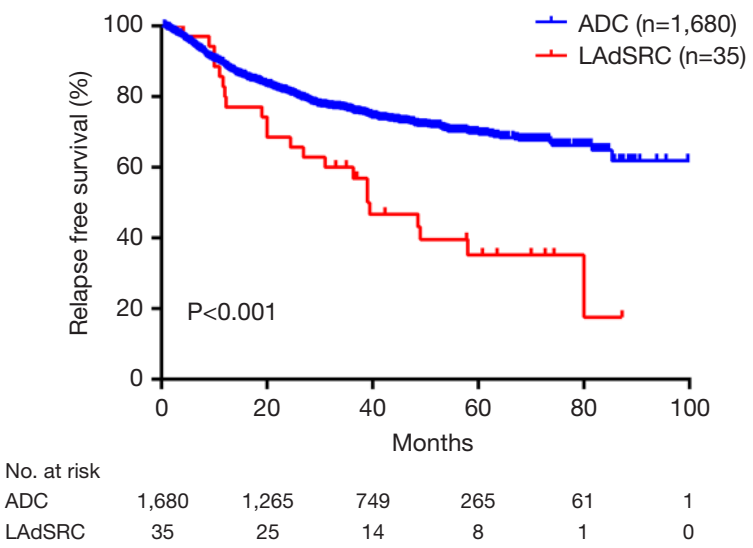

Figure 2 Kaplan-Meier survival curves for OS according to LAdSRCs and lung ADCs. OS, overall survival; LAdSRC, lung adenocarcinoma with signet ring cell; ADC, adenocarcinoma.

can be used as a marker to differentiate these tumors from malignant tumors that appear to be similar to those found in other organs $(11,23,24)$. Merchant et al. (16) indicated that TTF-1 was expressed in a high percentage of primary LAdSRCs and was very specific and that TTF-1 would be extremely valuable in distinguishing primary LAdSRCs from those arising in other organs. In that study, $82.4 \%$ of the 17 LAdSRCs expressed TTF-1 positivity, but no nonpulmonary SRCCs was positive for TTF-1, which was in line with our research results.

The immunostaining profiles of CK7 and CK20 also played an important role in distinguishing SRC from different organ sources $(13,14)$. From these previous studies, it was found that the CK profile of almost all breast SRCCs was expressed as CK7+/CK20-. In contrast, almost all colonic SRCCs showed an inverse pattern of immunostaining CK7-/CK20+. In the present study, 35 primary LAdSRCs showed the same high expression rate (69.7\%) as breast SRCCs in CK7+/CK20-. These results suggest that the CK7+/CK20 - mode favors primary LAdSRCs or breast SRCCs, whereas the CK7-/CK20+ pattern favors colonic origin. Villin is a protein that specifically binds to the actin core bundle of microvilli. Under normal physiological conditions, villus protein is mainly expressed in the digestive tract epithelial cells, renal proximal tubules and hepatobiliary ducts, and was not expressed in the lung $(25,26)$, which was consistent with our results, we didn't identify any villin $(+)$ in these 33 cases. However, Merchant et al. (16) have revealed the expression of villin in primary LAdSRCs recently, villin (+) was identified in 5 (29.4\%) of the 17 primary LAdSRCs in his study. The role of villin in identifying the source of SRC remain to be further studied.

There are several limitations in this study. First, the sample size of LAdSRCs was relatively small, so there may produce a bias. Second, those clinical and pathological data were retrospective in nature, and they should be confirmed in prospective trials if possible.

In summary, primary LAdSRC is a rare ADC subtype with a poor prognosis compared with ADC. The high percentage expression of TTF-1 and immunostaining profiles $\mathrm{CK} 7+/ \mathrm{CK} 20$ - in primary LAdSRC was significant to identify the source of SRCCs.

\section{Acknowledgments}

Funding: This work was supported by the National Natural Science Foundation of China (81773007); Outstanding youth program by Shanghai Municipal Commission of Health and Family Planning (2017YQ018); Shanghai Municipal Health and Family Planning Commission (201640213); Clinical Ancillary Department Capacity Building Project of Shanghai Shenkang Hospital Development Center (SHDC22015020).

\section{Footnote}

Conflicts of Interest: All authors have completed the ICMJE uniform disclosure form (available at http://dx.doi. org/10.21037/tcr.2019.11.54). The authors have no conflicts of interest to declare. 
Ethical Statement: The authors are accountable for all aspects of the work in ensuring that questions related to the accuracy or integrity of any part of the work are appropriately investigated and resolved. The study was conducted in accordance with the Declaration of Helsinki (as revised in 2013). The study was approved by the Institutional Review Board of Shanghai Chest Hospital $[\mathrm{KS}(\mathrm{Y}) 1668]$ and written informed consent was obtained from all patients.

Open Access Statement: This is an Open Access article distributed in accordance with the Creative Commons Attribution-NonCommercial-NoDerivs 4.0 International License (CC BY-NC-ND 4.0), which permits the noncommercial replication and distribution of the article with the strict proviso that no changes or edits are made and the original work is properly cited (including links to both the formal publication through the relevant DOI and the license). See: https://creativecommons.org/licenses/by-ncnd $/ 4.0 /$.

\section{References}

1. World Health Organization. Population fact sheets. Available online: http://globocan.iarc.fr/Pages/fact_sheets_ population.aspx

2. Travis WD, Brambilla E, Noguchi M, et al. International association for the study of lung cancer/american thoracic society/european respiratory society international multidisciplinary classification of lung adenocarcinoma. J Thorac Oncol 2011;6:244-85.

3. Devesa SS, Bray F, Vizcaino AP, et al. International lung cancer trends by histologic type: male:female differences diminishing and adenocarcinoma rates rising. Int $\mathrm{J}$ Cancer 2005;117:294-9.

4. Kish JK, Ro JY, Ayala AG, et al. Primary mucinous adenocarcinoma of the lung with signet-ring cells: a histochemical comparison with signet-ring cell carcinomas of other sites. Hum Pathol 1989;20:1097-102.

5. Colby TV, Noguchi M, Henschke C. Adenocarcinoma. In: Travis WD, Brambilla E, Müller-Hermelink HK, et al. editors. WHO classification of tumours of the lung, pleura, thymus and heart. Lyon: IARC Press, 2004.

6. Travis WD, Yatabe Y, Brambilla E, et al. Variants of adenocarcinoma. In: Travis WD, Brambilla E, Burke AP, et al. editors. WHO classification of tumours of the lung, pleura, thymus and heart. 4th ed. Lyon: IARC Press; 2015.

7. Tsuta K, Ishii G, Yoh K, et al. Primary lung carcinoma with signet-ring cell carcinoma components: clinicopathological analysis of 39 cases. Am J Surg Pathol 2004;28:868-74.

8. Ou SH, Ziogas A, Zell JA. Primary signet-ring carcinoma (SRC) of the lung: a population-based epidemiologic study of 262 cases with comparison to adenocarcinoma of the lung. J Thorac Oncol 2010;5:420-7.

9. Cohen PR, Yoshizawa A, Motoi N, et al. Signet ring cell features (SRCF) in lung adenocarcinoma: a cytologic feature or a histologic subtype? Mod Pathol 2010;23:400A.

10. Hayashi H, Kitamura H, Nakatani $Y$, et al. Primary signet-ring cell carcinoma of the lung: histochemical and immunohistochemical characterization. Hum Pathol 1999;30:378-83.

11. Fabbro D, Di Loreto C, Stamerra O, et al. TTF-1 gene expression in human lung tumours. Eur J Cancer 1996;32A:512-7.

12. Folpe AL, Gown AM, Lamps LW, et al. Thyroid transcription factor-1: immunohistochemical evaluation in pulmonary neuroendocrine tumors. Mod Pathol 1999;12:5-8.

13. Goldstein NS, Long A, Kuan SF, et al. Colon signet ring cell adenocarcinoma: immunohistochemical characterization and comparison with gastric and typical colon adenocarcinomas. Appl Immunohistochem Mol Morphol 2000;8:183-8.

14. Tot $\mathrm{T}$. The role of cytokeratins 20 and 7 and estrogen receptor analysis in separation of metastatic lobular carcinoma of the breast and metastatic signet ring cell carcinoma of the gastrointestinal tract. APMIS 2000;108:467-72.

15. Castro CY, Moran CA, Flieder DG, et al. Primary signet ring cell adenocarcinomas of the lung: a clinicopathological study of 15 cases. Histopathology 2001;39:397-401.

16. Merchant SH, Amin MB, Tamboli P, et al. Primary signet-ring cell carcinoma of lung: immunohistochemical study and comparison with non-pulmonary signet-ring cell carcinomas. Am J Surg Pathol 2001;25:1515-9.

17. Iwasaki $T$, Ohta $M$, Lefor AT, et al. Signet-ring cell carcinoma component in primary lung adenocarcinoma: potential prognostic factor. Histopathology 2008;52:639-40.

18. Butala RM, Moscovic EA. Neuroendocrine markers in pulmonary adenocarcinomas with signet-ring cells. Hum Pathol 1990;21:1082.

19. Hiraki A, Ueoka H, Yoshino T, et al. Primary signetring cell carcinoma of the lung with histochemical characterization. Anticancer Res 2002;22:1079-81.

20. Huber M, Pavlova B, Mühlberger H, et al. Detection of the 
Epstein-Barr virus in primary adenocarcinoma of the lung with Signet-ring cells. Virchows Arch 2002;441:25-30.

21. Maemondo M, Saijo Y, Ebina M, et al. Rapidly progressive primary adenocarcinoma of the lung with signet-ring cells responding to 5-fluorouracil and leucovorin: A case report. Int J Clin Oncol 1997;2:47-50.

22. Whitsett JA, Glasser SW. Regulation of surfactant protein gene transcription. Biochim Biophys Acta 1998;1408:303-11.

23. Di Loreto C, Di Lauro V, Puglisi F, et al. Immunocytochemical expression of tissue specific transcription factor-1 in lung carcinoma. J Clin Pathol

Cite this article as: Chen C, Wang L, Gu C, Wang Y, Pan X, Fu S, Yang J, Wang R. Survival analyses and immunohistochemical study of primary signet ring cell carcinoma of the lung adenocarcinoma. Transl Cancer Res 2020;9(2):620-628. doi: 10.21037/tcr.2019.11.54
1997;50:30-2.

24. Fabbro D, Di Loreto C, Beltrami CA, et al. Expression of thyroid-specific transcription factors TTF-1 and PAX-8 in human thyroid neoplasms. Cancer Res 1994;54:4744-9.

25. Bacchi CE, Gown AM. Distribution and pattern of expression of villin, a gastrointestinal-associated cytoskeletal protein, in human carcinomas: a study employing paraffin-embedded tissue. Lab Invest 1991;64:418-24.

26. Robine S, Huet C, Moll R, et al. Can villin be used to identify malignant and undifferentiated normal digestive epithelial cells? Proc Natl Acad Sci U S A 1985;82:8488-92. 


\section{Supplementary}

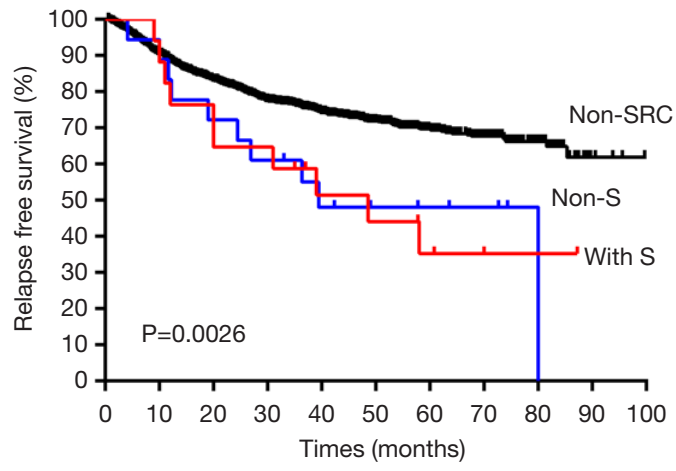

Figure S1 Kaplan-Meier survival curves for RFS based on whether LAdSRCs contained solid components. RFS, relapse-free survival; LAdSRC, lung adenocarcinoma with signet ring cell; S, solid component.

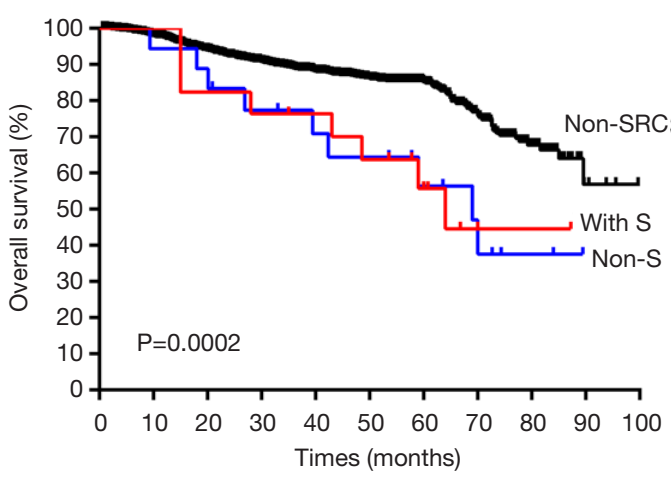

Figure S2 Kaplan-Meier survival curves for OS based on whether LAdSRCs contained solid components. OS, overall survival; LAdSRC, lung adenocarcinoma with signet ring cell; S, solid component. 